\title{
Deformation and fracture characteristics of zirconium plate produced via ultrasonic additive manufacturing
}

\author{
Caleb P. Massey ${ }^{1, a)} @$, Nitish Bibhanshu ${ }^{1}$, Maxim N. Gussev ${ }^{1}$, Cody J. Havrilak ${ }^{1}$, \\ Andrew T. Nelson ${ }^{1}$ \\ ${ }^{1}$ Oak Ridge National Laboratory, Oak Ridge, TN 37831, USA \\ a) Address all correspondence to this author. e-mail: masseycp@ornl.gov
}

Received: 2 June 2021; accepted: 2 September 2021; published online: 28 October 2021

This manuscript has been authored by UT-Battelle, LLC, under contract DE-AC05-00OR22725 with the US Department of Energy (DOE). The US government retains and the publisher, by accepting the article for publication, acknowledges that the US government retains a nonexclusive, paid-up, irrevocable, worldwide license to publish or reproduce the published form of this manuscript, or allow others to do so, for US government purposes. DOE will provide public access to these results of federally sponsored research in accordance with the DOE Public Access Plan (http://energy.gov/ downloads/doe-public-access-plan).

The microstructural evolution, deformation modes, and fracture mechanisms of zirconium plate produced using ultrasonic additive manufacturing (UAM) are presented. In addition to conventional tensile testing techniques, digital image correlation captured highly variable strain accumulation in specimens loaded perpendicular or parallel to the build height $(Z)$. When tested in parallel to $Z$, delamination at prior foil/foil interfaces creates strain localization noticeable in strain rate maps, whereas specimens loaded perpendicular to $Z$ illustrate conventional strain hardening until necking accelerates delamination. Although bond strengths are statistically and spatially variable, in situ electron backscattering diffraction tests illustrate the ability for grains near interfaces to accommodate strain with twinning and slip modes consistent with conventionally produced zirconium alloys. Finally, mixtures of ductile and delamination-induced fracture highlight the interface-driven failure modes of UAM zirconium plate in the as-built condition.

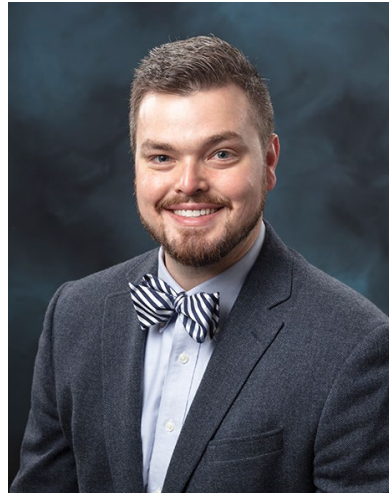

Caleb P. Massey
Caleb P. Massey is a staff member in the Advanced Fuel Fabrication and Instrumentation Group at Oak Ridge National Laboratory. His research focuses on irradiation effects on materials, with a strong emphasis on advanced fuel and cladding systems for nuclear reactors. Caleb received his Bachelor's and Master's degrees in Mechanical Engineering from Virginia Commonwealth University in Richmond, VA. He then went on to receive his Ph.D. in Nuclear Engineering from the University of Tennessee in Knoxville, TN. During his Ph.D. studies, his research focused on the evaluation of accident tolerant fuel cladding concepts designed for enhanced oxidation resistance during nuclear accident scenarios. This work evolved into an optimization of thermomechanical processing parameters used in the production of a special class of ironbased alloys known as nanostructured ferritic alloys. In 2019, Caleb was hired at Oak Ridge National Laboratory, where he has continued his involvement with various materials development, irradiation, post-irradiation examination, and qualification efforts. In addition, he is the point-of-contact for ultrasonic additive manufacturing efforts at the lab for applications such as sensor embedding and composites manufacturing. 


\section{Introduction}

Ultrasonic additive manufacturing (UAM) is a joining technique that uses multiple sequential ultrasonic foil welds to build larger layered 3D structures. The UAM technique was first patented in 1999 and has rapidly grown in popularity because of the expanded design space allowed by the solidstate and lower-temperature nature of the technique $[1,2]$. Joining via the UAM process is enabled by the use of an ultrasonic transducer that is connected to a cylindrically shaped workpiece called a "sonotrode." The harmonic oscillations induced by the transducer force the sonotrode to vibrate at a $20 \mathrm{kHz}$ frequency, thereby creating a scrubbing motion atop the foil to be welded. This scrubbing motion, coupled with an applied force normal to the surface of the foil, creates significant shear stresses that dissolve intermediate oxide layers and promote contact between the metallic surfaces underneath [3].

Because of its relatively low temperature of operation $(<0.5$ $T_{\mathrm{M}}$, where $T_{\mathrm{M}}$ is the melting point of the material being welded), coupled with its in situ subtractive machining capabilities that can be implemented to create parts of complex geometry, the technique has become a popular method for applications such as sensor embedding [4, 5] and composite manufacturing [6-8]. The ability of UAM to create spatially engineered composites has recently been demonstrated for the nuclear field through the production of prototype composite control plates for Oak Ridge National Laboratory's High Flux Isotope Reactor [9].

In an effort to broaden UAM's application space in the nuclear field, the technique has recently been applied for the first time to zirconium ( $\mathrm{Zr}$ ), a popular nuclear structural material because of its good balance of corrosion resistance, strength, and low neutron absorption cross section. The ability to create $\mathrm{Zr}$ structures using the UAM technique potentially opens new

TABLE 1: Tensile properties of individual UAM Zr SST specimens, including the yield strength (YS), ultimate tensile strength (UTS), uniform elongation (UE), and total elongation (TE).

\begin{tabular}{lrrrrr}
\hline \hline Orientation & Specimen ID & YS [MPa] & UTS [MPa] & UE [\%] & TE [\%] \\
\hline X & AM-X1 & 341 & 415 & 7.7 & 40.5 \\
& AM-X2 & 378 & 441 & 5.4 & 33.7 \\
& AM-X3 & 354 & 442 & 8.3 & 37.0 \\
& AM-X4 & 368 & 441 & 5.3 & 27.7 \\
Y & AM-Y1 & 345 & 430 & 7.1 & 41.3 \\
& AM-Y2 & 398 & 471 & 6.2 & 39.0 \\
& AM-Y3 & 355 & 473 & 7.3 & 34.4 \\
& AM-Y4 & 346 & 452 & 10.7 & 39.5 \\
& AM-Z1 & 380 & 473 & 7.1 & 39.7 \\
& AM-Z2 & 183 & 183 & 0.0 & 0.0 \\
& AM-Z3 & 370 & 405 & 0.7 & 15.6 \\
& AM-Z4 & 363 & 376 & 0.5 & 11.4 \\
\hline \hline
\end{tabular}

design spaces for in situ monitoring or even composite fuel concepts. In preliminary microstructure and mechanical property investigations of a newly fabricated UAM Zr plate, the strength of the build was competitive with that of commercial grade $\mathrm{Zr}$ plate; but it exhibited highly stochastic ductility and strength when loaded parallel to the build direction [10]. Thus, this work aims to explain the previously observed anisotropic mechanical properties through complementary in situ tensile characterization techniques, namely digital image correlation (DIC) and electron backscatter diffraction (EBSD).

\section{Experimental results and discussion}

\section{DIC tensile response of UAM Zr specimens}

A summary of individual specimen tensile statistics is provided in Table 1. Generally, the specimens tested in the $\mathrm{X}$ (sonotrode travel) and Y (vibration) orientations behaved similarly, with uniform elongations between 5 and $7 \%$ as measured in engineering strain. The strengths of these specimens were much higher than was expected for commercial grade Zr. According to the ASTM standard for Zr plate (ASTM B551), the yield strength for unalloyed $\mathrm{Zr}$ must be at least $205 \mathrm{MPa}$, with a corresponding ultimate tensile strength of at least $380 \mathrm{MPa}$ [11]. For this analysis of first-generation UAM Zr, the average yield strength (YS) and ultimate tensile strength (UTS) in all orientations exceeded those of the ASTM standard. This increase in strength was primarily due to the significant work hardening, twinning, and grain refinement of the $\mathrm{Zr}$ microstructure associated with the compressive loads and shear stresses in the UAM process.

Major differences in strength, ductility, and reproducibility existed in the tensile data as a function of specimen orientation. Since UAM welds sequential layers repeatedly to add specimen height, specimens tested parallel to the build height (Z-direction) had gauge sections comprising multiple weld layers compared with the $\sim$ three layers across the gauge sections in $\mathrm{X}$ and $\mathrm{Y}$ specimens. In some cases, critical weld defects (as in the AM-Z2 specimen) resulted in the brittle failure of the specimen under initial loading in the elastic region. In the AM-Z1 specimen, the lack of weld defects led to similar mechanical properties to those loaded in the $\mathrm{X}$ and $\mathrm{Y}$ orientations, which has been highlighted in a previous work [10]. Most specimens, however, exhibited more complex behavior. An example of such behavior is exemplified by the AM-Z3 and AM-Z4 specimens, which were able to reach yield before an immediate transition into a region of strain instability.

The difference in plastic behavior between specimens loaded parallel and perpendicular to the build height is shown using strain rate maps for representative specimens AM-Y3 and AM-Z3 in Fig. 1. Strain rate maps are effective tools for visualizing deformation mechanisms over the entire gauge region. 


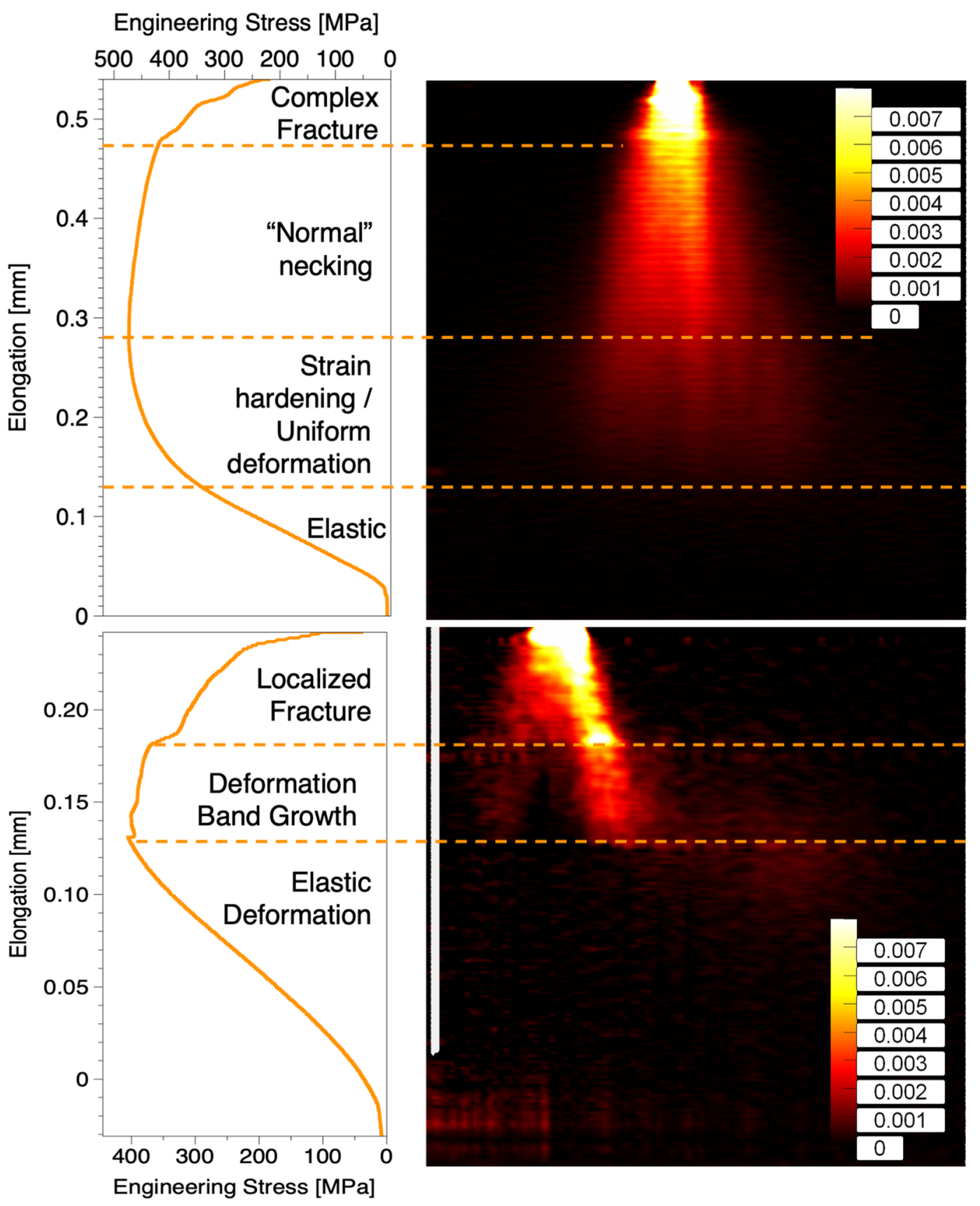

Figure 1: Von Mises strain rate maps for $\mathrm{AM}-\mathrm{Y} 3$ (top) and AM-Z3 (bottom) specimens. The $\mathrm{X}$-axis shows the position along the gauge, and the $\mathrm{Y}$-axis denotes the time (elongation/strain) at which the measurement is made. The map is color coded so that nominal strains $\left(\dot{\varepsilon} \sim 0.001 \mathrm{~s}^{-1}\right)$ appear in dark red, deformation bands $\left(0.001<\dot{\varepsilon}<0.007 \mathrm{~s}^{-1}\right)$ in red, and strain localization/necking $\left(\dot{\varepsilon}>0.007 \mathrm{~s}^{-1}\right)$ in yellow/white.

Each X-value on the strain rate plot corresponds to the distance across the tensile gauge length, while Y-values indicate the time (or equivalently the distance along the tensile curve) associated with the data point. Thus, the thermal map of strain rates provides a spatially and temporally resolved visualization of how the specimens deformed over the course of the tensile test. Along the Y-axis of each plot, the corresponding tensile curve is also plotted to show the characteristic deformation regions.
For the AM-Y3 specimen, the strain rate map demonstrates early deformation behavior characteristic of standard plasticity in the elastic and strain hardening regimes. In the elastic region, as would be expected, no plastic deformation is registered through the DIC analysis, indicated by the black color in the plot. Similarly, the very ends of the $\mathrm{X}$-axis in all regimes are colored black, showing that little to no deformation is occurring in the grips/head region of the SST specimen. That result 


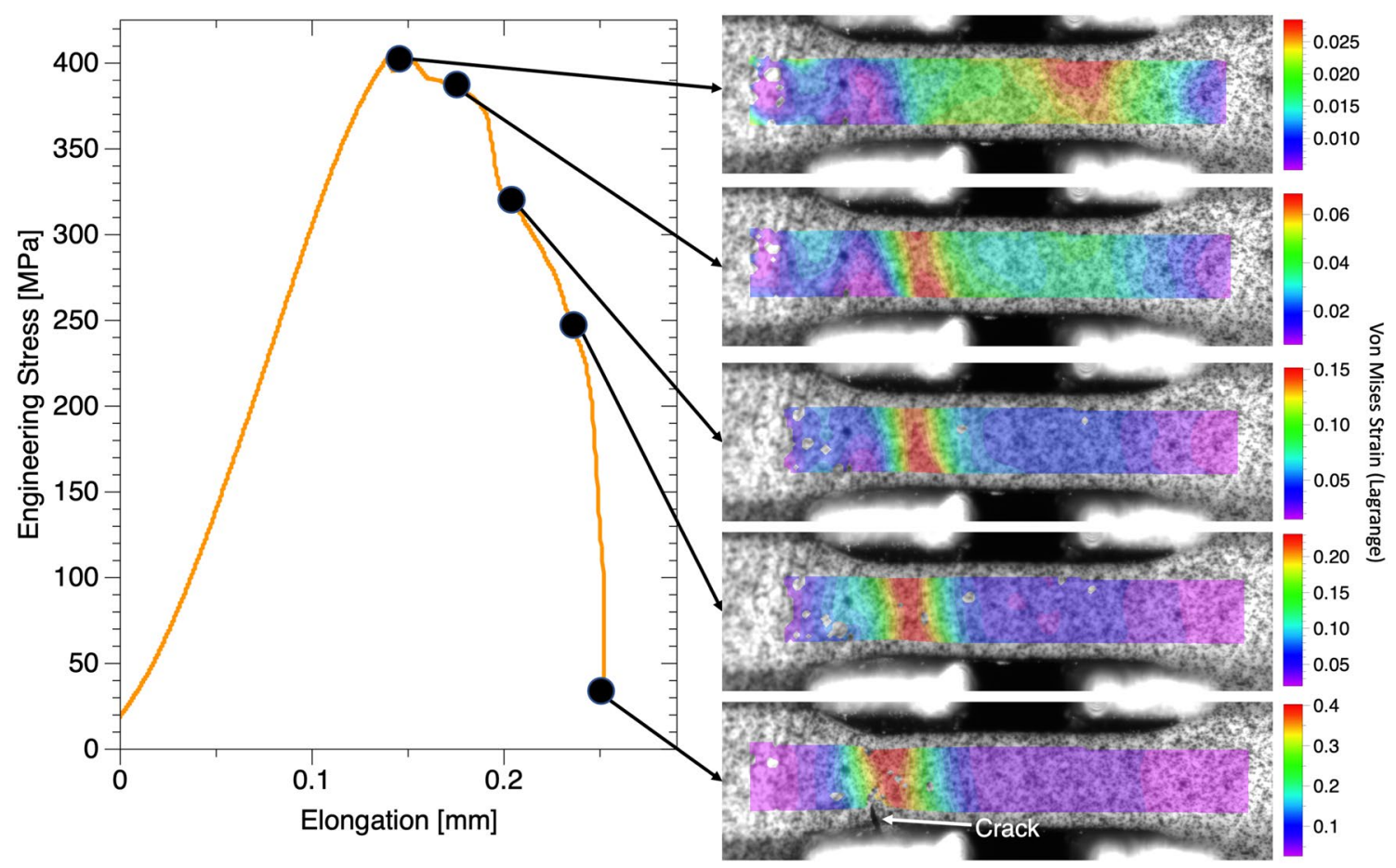

Figure 2: Von Mises strain maps for the AM-Z3 specimen at five different locations along the tensile curve. Strain maps are overlaid against corresponding DIC optical images for each condition.

provides additional confidence in the miniature specimen geometry. In the uniform deformation region of the tensile curve, between the YS and the UTS values, the gauge section begins to deform homogeneously with only slight indications of vertical dislocation bands throughout the gauge area. After exceeding the UTS, the triangular-shaped narrowing and intensification of the strain rate toward the center of the tensile specimen highlights the conventional loss of plastic stability and necking as material flow reduces the local cross sectional area of the gauge center. Fracture in the AM-Y3 specimen shows some abnormality in the form of local strain "bursts." These strain bursts, indicated by horizontal flashes of higher strain rate intensities, correspond to the fluctuating and wavy tensile curve shape as the specimen fractures in a more complex way. This complex fracture is revisited and explained in "Microstructural evolution of UAM Zr using in situ EBSD” section.

For specimen AM-Z3, the strain rate map shows similar behavior up to the YS of its tensile curve. The initial dark red indications of strain that occur at loads $<10 \mathrm{MPa}$ are due to artifacts associated with the specimen shifting initially in the grips under loading. Similarly to the Y-oriented specimen, the $\mathrm{Z}$ specimen shows no indication of accumulating strain during the initial loading to the YS. After exceeding the YS, the behavior becomes quite complex. Thus, in addition to the strain rate map, Von Mises strain maps are overlaid atop the optical DIC images at characteristic locations along the AM-Z3 tensile curve in Fig. 2. The initiation of plastic strain accumulation at the YS concentrates as a weak deformation band on the right-hand portion of the tensile gauge, which accumulates only $2.5 \%$ local plastic strain. A major deformation band quickly develops after this point just to the left of the gauge center, which is emphasized by the rapid increase in strain rate over a small area in Fig. 1 between the dotted lines. Whereas the remainder of the gauge section fails to achieve more than $5 \%$ strain over the remaining duration of the tensile test, this region of significant deformation rapidly accumulates $\sim 40 \%$ local plastic strain as the specimen loses load-bearing capacity and eventually fractures, as is shown by the initiation of a crack on the bottom portion of the tensile specimen in the bottom image of Fig. 2. This large deformation band is likely a delaminated foil/foil boundary, as this type of failure is characteristic of prior research on lower-strength UAM materials such as aluminum [12].

\section{Microstructural evolution of UAM Zr using in situ EBSD}

In the realm of UAM, hypotheses explaining the non-ideal properties of materials in the build direction have usually fallen into two primary categories: insufficient layer adhesion and insufficient post-weld foil plasticity. In the first case, some researchers have proposed that poor bonding has been due to either the presence of voids at interfaces [3] or insufficient layer-to-layer metallurgical bonds [13]. Others have hypothesized that strain accommodation at interfaces is reduced as a result of either UAM-induced texturing and/or high preexisting strains in UAM 

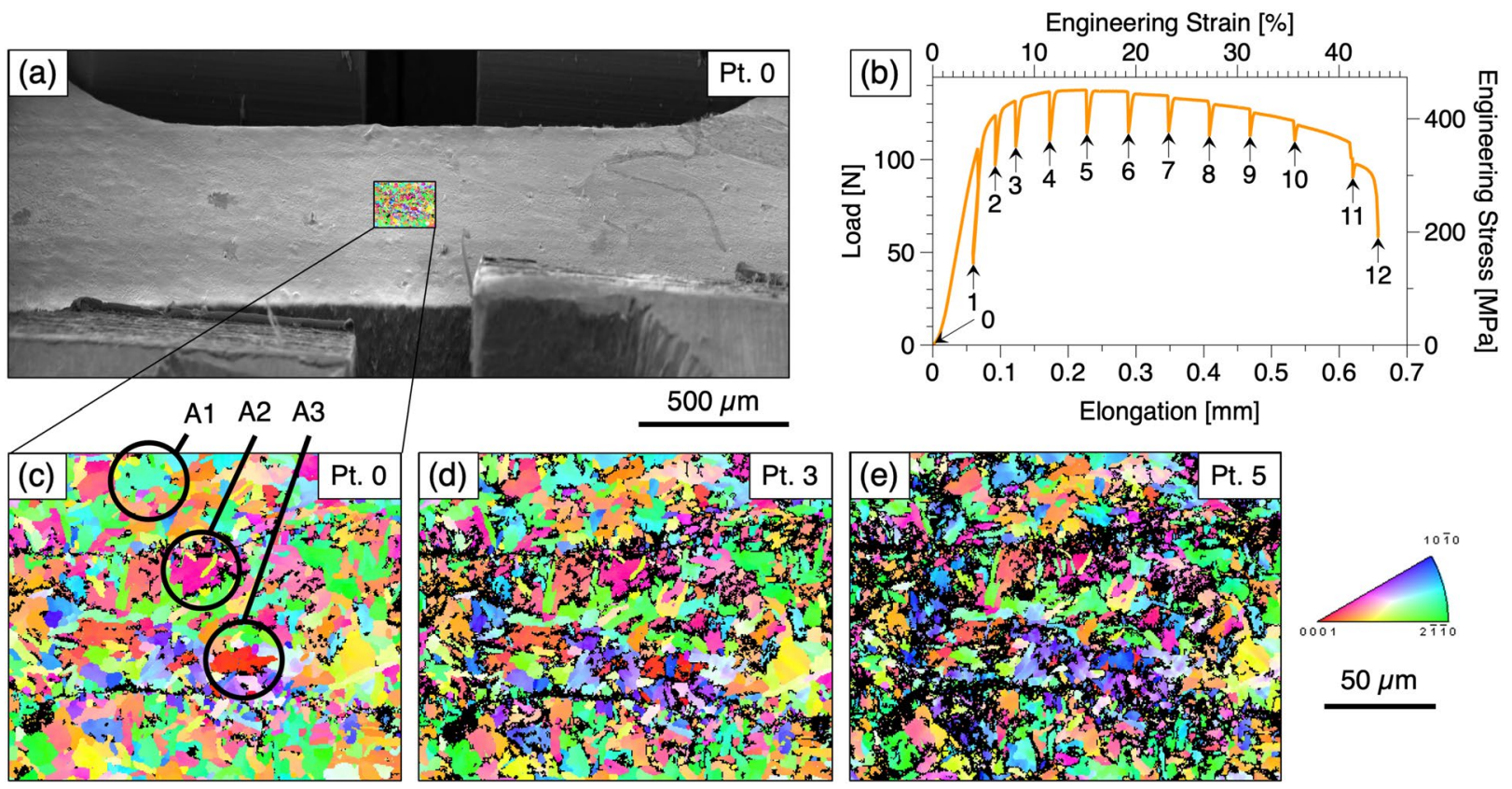

Figure 3: In situ EBSD results for AM-Y4. In (a) the unstrained gauge section is shown in a secondary electron image held by the two SST grips. In (b), the engineering stress/strain (load/elongation) tensile curve is provided with numbered locations at which load was released for image acquisition. EBSD IPF maps for select locations show the grain orientation evolution between the (c) unloaded condition, (d) shortly after yielding, and (e) the ultimate tensile strain. All inverse pole figure maps are oriented so their plane normal is parallel to the tensile axis.

builds [14]. Using in situ tensile SEM/EBSD, these hypotheses are compared against a specimen loaded perpendicular to the build direction (AM-Y4 in Table 1).

An SEM micrograph of the tensile specimen prior to loading is shown in Fig. 3a. Also visible and obstructing a small portion of the gauge are the SST grips used in the EBSD tensile setup. In Fig. 3b, the tensile curve is shown with various numbered points corresponding to the inverse pole figure (IPF) maps in Fig. 3c-e. Although the tensile specimen is viewed in the SEM image along the $\mathrm{YZ}$ plane with respect to the sample coordinate system, the IPF maps are colored with respect to the tensile axis to simplify the visualization of various slip and twinning systems. In this specimen, five individual $\mathrm{Zr}$ foil layers exist across the gauge width, and three of these are captured in the EBSD maps in Fig. 3. The interfaces between the three layers are visible because of the low confidence of indexed points at these boundaries.

The as-manufactured grain structure is highly deformed with clear evidence of significant preexisting twin structures. Previous work has shown that $\{10 \overline{1} 1\}$-type twins dominate in the as-manufactured UAM grain structure, a result of the relatively high friction-induced temperature and compressive loading reached at the interfaces during the UAM process [10]. One interesting observation is that, aside from the visible layer interfaces in the EBSD maps, the interfaces are difficult to identify if they are based only on visible differences in grain deformation behavior close to and away from these boundaries. In fact, the grain structure appears similar near the centerline of the foil as it does near the interfaces, suggesting that the entire foil experiences significant deformation during the UAM process, and that strains are not localized solely at interfacial regions.

The EBSD analysis also shows that the as-deformed grain structure both near and away from the prior foil/foil interfaces can readily accommodate strains through the available slip systems in hexagonal-close-packed (HCP) Zr. In Fig. 3c, three different areas (A1, A2 and A3) are highlighted and extracted to track their individual grain evolution over the course of the tensile testing (Fig. 4). Area 1 has a $\sim 15^{\circ}$ misorientation with respect to $(2 \overline{11} 0)$ prior to the tensile test and is located $\sim 50 \mu \mathrm{m}$ away from the topmost interface visible in the EBSD map. During deformation, this grain slowly changes orientation towards $(10 \overline{1} 0)$ via slip. It is well-known that prismatic $\{10 \overline{1} 0\}\langle 11 \overline{2} 0\rangle$ slip has been established as the easiest dislocation glide mode in $\mathrm{Zr}$ $[15,16]$, followed by pyramidal $\{10 \overline{1} 1\}\langle 11 \overline{2} 3\rangle$ and then basal $\{0001\}\langle\overline{11} 20\rangle[17,18]$. Although increased strain rates and test temperatures can fundamentally change the active slip systems [19], this test was conducted at a low strain rate $\left(10^{-3} \mathrm{~s}^{-1}\right)$ and at room temperature, so it is unsurprising that the results here are consistent with the prior literature.

Two other select regions of differing orientations are shown in Fig. 4. One of the regions, oriented close to (1013), also borders prior twin boundaries with orientations ranging from $57^{\circ}$ to $59^{\circ}$. The two primary c-axis contraction (compression) twins available for $\mathrm{Zr}$ are the $\{11 \overline{2} 2\}\langle 11 \overline{23}\rangle$ twin, which reorients the lattice $64.22^{\circ}$ about $\langle 10 \overline{1} 0\rangle$, and the $\{10 \overline{1} 1\}\langle 10 \overline{12}\rangle$ twin, which 

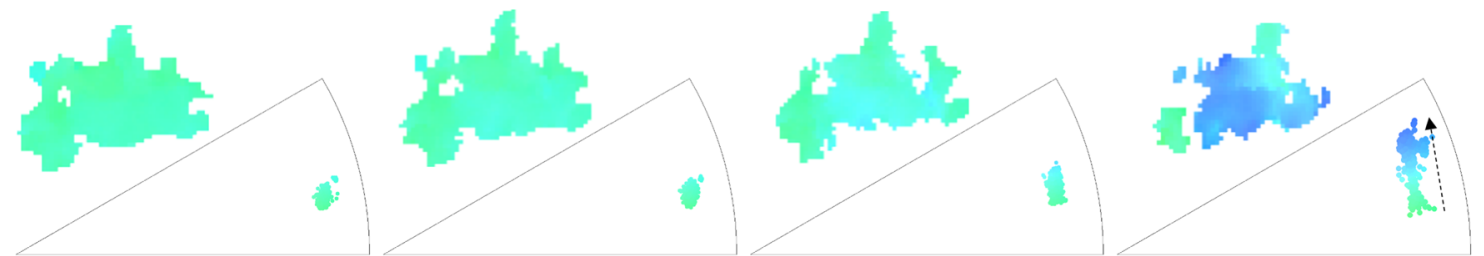

Pt. 0

Pt. 2

Pt. 3

Pt. 5

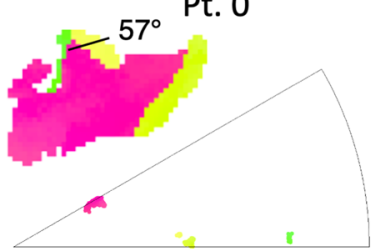

Pt. 0

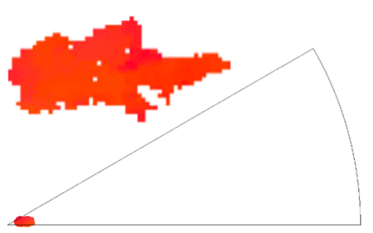

Pt. 0

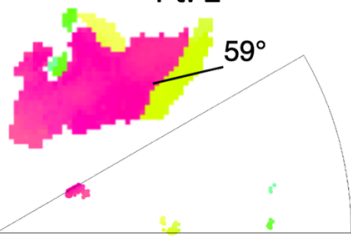

Pt. 2

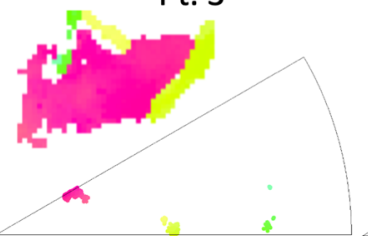

Pt. 3

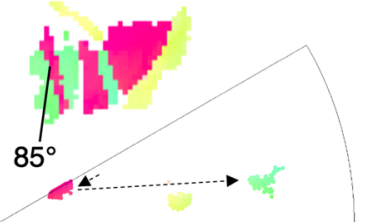

Pt. 5

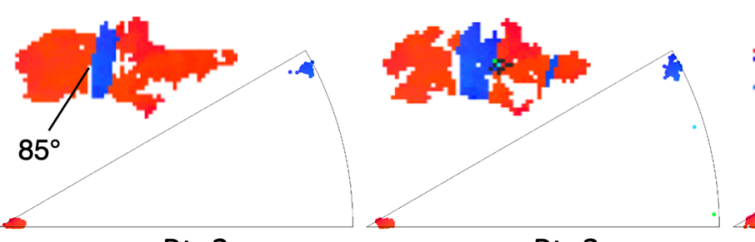

Pt. 2

Pt. 3

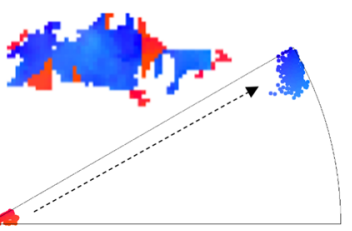

Pt. 5

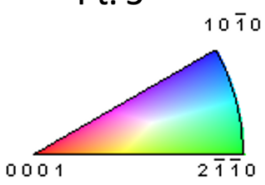

Figure 4: The microstructural evolution of three local areas/grain(s) is shown using inverse pole figures. Areas correspond to $A 1, A 2$, and $A 3$ as labeled in Fig. 3. All inverse pole figures are oriented parallel to the tensile axis. Labeled points indicate the scan location according to the in situ EBSD tensile curve.

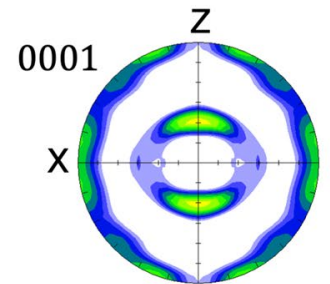

Z
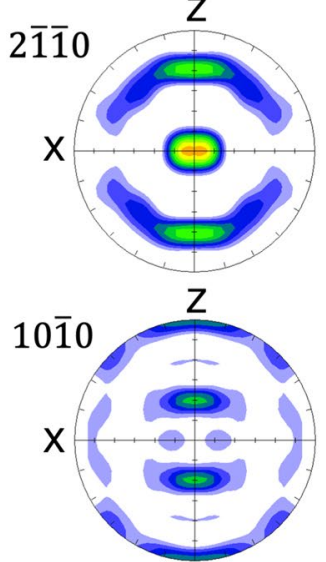

(a) Pt. 0

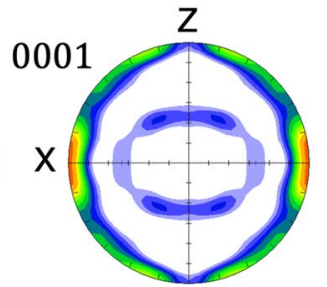

Z

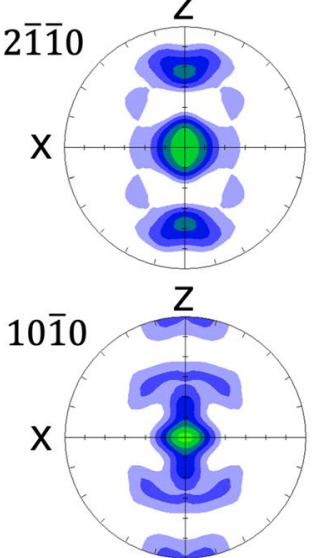

(b) Pt. 6
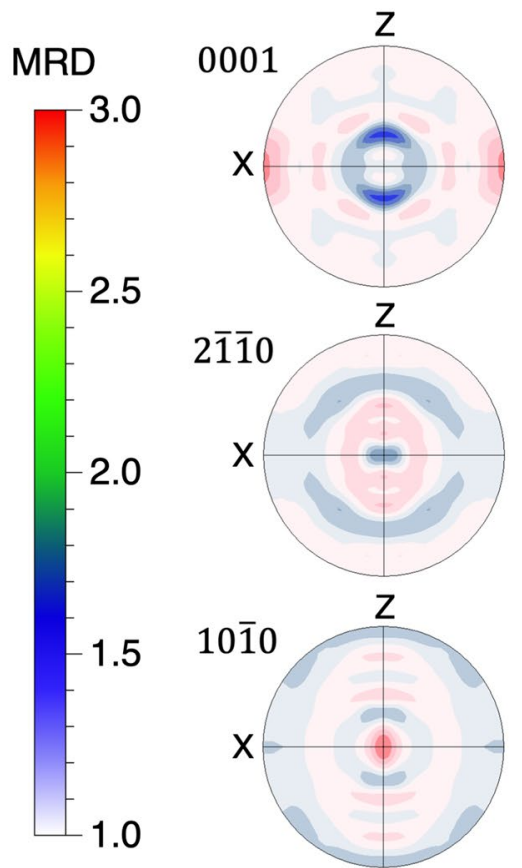

MRD

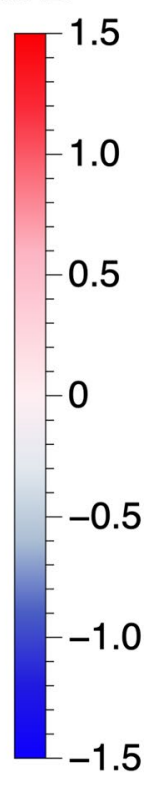

(c) Difference

Figure 5: The evolution of the microstructural texture along the gauge section over the course of the in situ EBSD tensile test. In (a) the three principal pole figures are provided for the unstrained condition, while (b) shows the texture at point 6 in the tensile curve, at the onset of necking. The difference between pole figures is plotted in (c), where intensifying components appear in red and weakening components are blue. Color bars use units of multiples of random density (MRD). 
reorients the lattice $57.05^{\circ}$ about $\langle 11 \overline{2} 0\rangle$ [20]. Thus, the preexisting twins are more likely the $\{10 \overline{1} 1\}$-type. Following deformation to point 5 on the tensile curve, corresponding to the UTS of the specimen, the parent grain begins deforming via tensile twinning $\{10 \overline{1} 2\}\langle 10 \overline{11}\rangle$, which is characterized by a rotation of $85.22^{\circ}$ about $\langle 10 \overline{2} 0\rangle$ [20]. Similar tensile twins are also noted for a grain with an initial (0001) orientation, with an almost complete twinning reorientation by the time the UTS is reached. Both of the latter grains are located along either of the visible foil/foil boundaries in Fig. 3, which provides evidence that the grains near/adjacent to foil interfaces still have the capability for significant deformation and reorientation. Even so, future studies of Z-orientation specimens with sufficient weld quality are needed to assess the textural hypothesis, as it is known that texture in $\mathrm{Zr}$ can have a significant influence on strain hardening and deformability [21].

The same trends seen in the individual grain data extend to the mesoscale texture trends as analyzed via the larger EBSD gauge scans. In Fig. 5, the pole figures at pt. 0 (prior to straining) are compared with those at point 6 , shortly after entering the region of plastic instability. The difference between the two plots (Fig. 5c) shows areas of increasing intensity in red and areas of weakening intensity in blue, with white indicating no change. The previously existing split basal texture decreases in intensity while the $\{10 \overline{1} 0\}\langle 11 \overline{2} 0\rangle$ texture components increase in both the $(10 \overline{10} 0)$ and $(2 \overline{11} 0)$ pole figures. In addition, much of the prior complex rolling texture diminishes, as tensile slip and twinning modes drive deformation behavior. These data further support the hypothesis that areas away from and near the interfaces can accommodate tensile strains through conventional slip and twinning mechanisms available to the $\mathrm{Zr}$ lattice.

Later in the tensile test, the same complex fracture characteristics previously seen in the AM-Y3 DIC specimen are seen in the AM-Y4 EBSD specimen. The reasons for this complex fracture arise from late-stage foil delamination, as illustrated in the SEM images of the necked gauge (Fig. 6). In this case, only one of the interfaces delaminated during the test, but its onset was rapid and agreed with the strain bursts seen previously in the DIC data. Future microscopy work will be performed to evaluate local microstructural differences between the surfaces
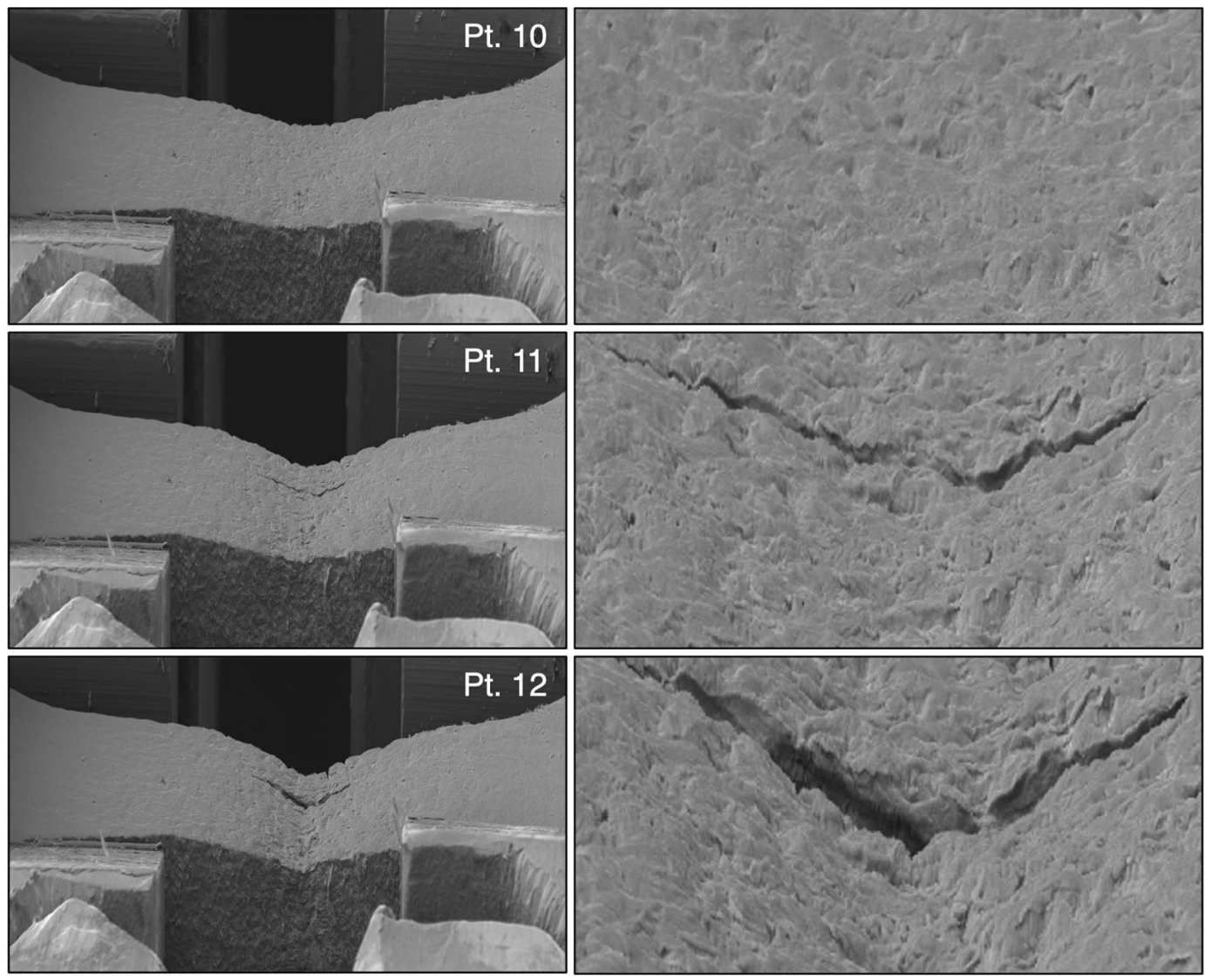

$1 \mathrm{~mm}$

Figure 6: Secondary electron SEM images of the AM-Y4 gauge section in the latter stages of mechanical instability and necking. In pt. 10, a clear reduction in thickness at the center of the gauge section is noticeable, but no surface cracking is observed. In pt. 11 and pt. 12 , a clear widening crack parallel to the tensile axis develops at a prior foil/foil boundary in the UAM Zr material. 

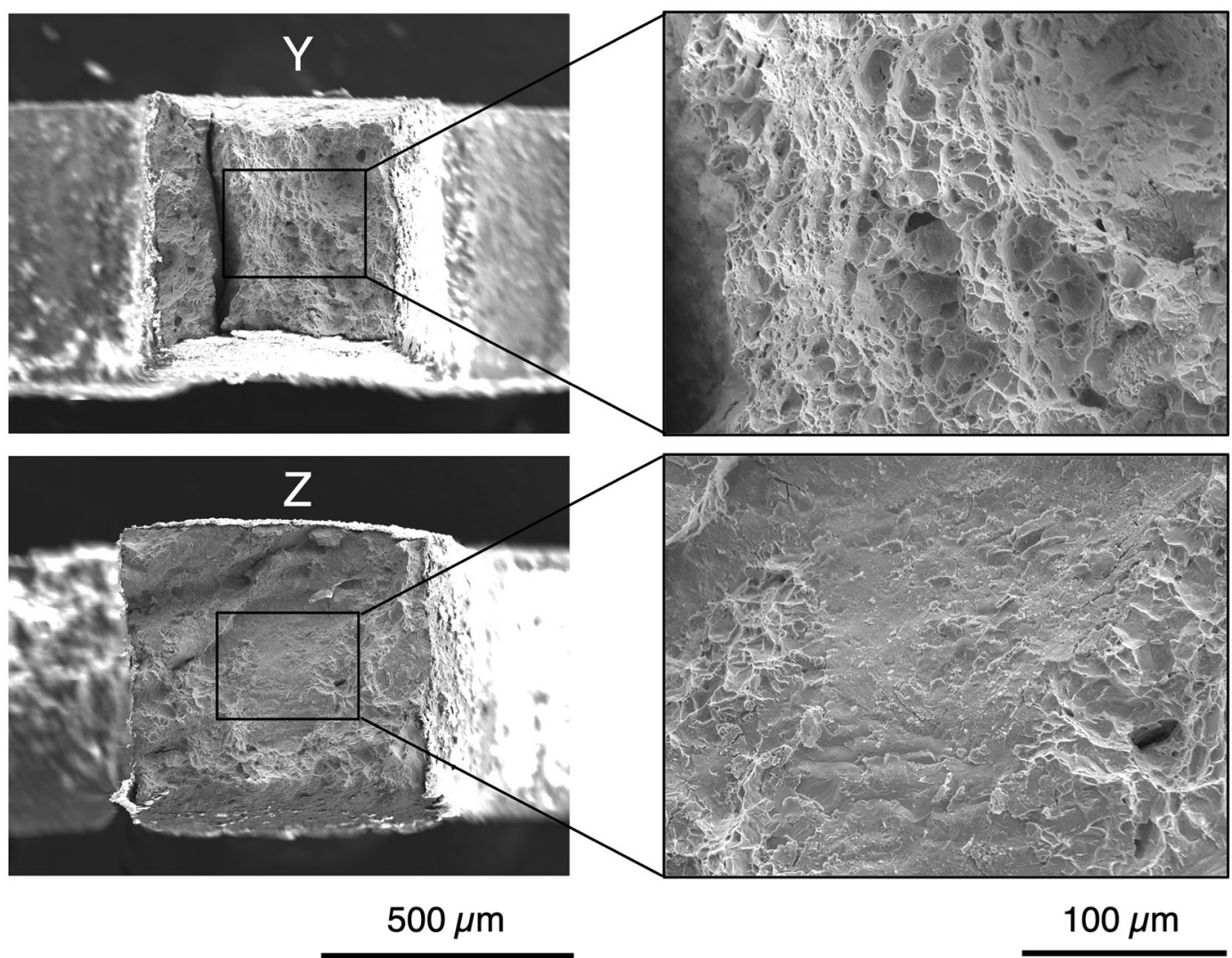

Figure 7: Secondary electron images of fracture surfaces of miniature tensile specimens AM-Y3 and AM-Z3. The Y specimen shows clear ductile dimpling and a foil/foil delamination. The Z-orientation specimen shows a mixture of ductile dimpling in select areas with evidence of brittle cleavage fracture across much of the sample surface.

that delaminated vs. those that retained their adhesion to the conclusion of the tensile test.

\section{Fractography informed failure modes}

Immediately following sample fracture, the fracture surfaces of select representative specimens were imaged to assess fracture modes. As with the DIC and EBSD tensile data, similar trends were seen for the specimens tested perpendicular (XY) and parallel (Z) to the build height orientation. For the DIC specimen AM-Y3 that showed complex fracture characteristics, most of the fracture surface exhibits ductile dimple behavior (Fig. 7). One large foil/foil delamination exists, and a careful examination of the inner surface shows a somewhat smooth surface characteristic of brittle cleavage/delamination, even after significant deformation strains. Thus it appears that, in the $\mathrm{X}$ and Y-oriented specimens, interface debonding did not occur until late in the tensile test because the uniaxial nature of the test allowed each individual foil to assume the load. This did not cause significant transverse stresses across the interface until necking-induced mass flow of each individual foil caused gaps to open between foil-foil interfaces.
For the Z-oriented specimen, AM-Z3, there are instances of ductile dimpling at the left and right sides of the enlarged image (Fig. 7). The remaining surface exhibits clear brittle fracture. Previous microstructural investigations have indicated that using a Ti-foil buffer layer during the UAM Zr process caused traces of Ti impurities to appear along certain foil-foil interfaces [10]. However, because of local topography changes along these fracture surfaces, energy dispersive $\mathrm{x}$-ray spectroscopy could not be used to resolve minor variations in possible $\mathrm{Ti}$ enrichment on the fracture surface. Also, there do not appear to be pockets of voids along the fractured interface, so weld quality and/or impurities, not the existence of porosity, may be the primary factor influencing Z-orientation deformation behavior in firstgeneration UAM Zr materials.

\section{Conclusions}

The deformation and fracture characteristics of first-generation UAM Zr builds were assessed using a combination of in situ DIC, EBSD tensile tests, and fractography. It was found that although microstructural evolution was similar to that of conventionally produced $\mathrm{Zr}$ alloys under tensile loading, the 


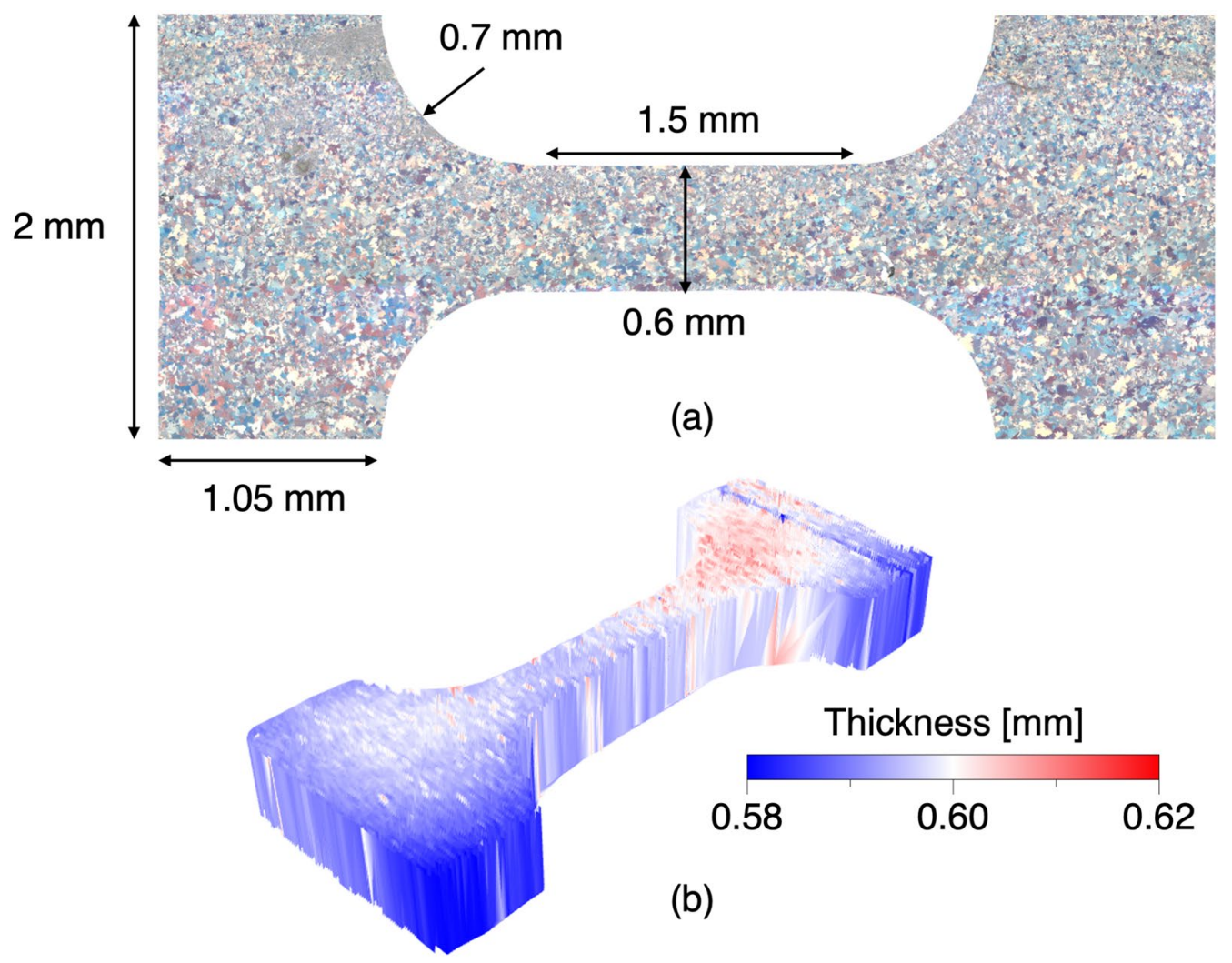

Figure 8: Representative SST specimen machined in the Y-direction (parallel to sonotrode vibration direction). In (a), a polarized light micrograph shows the fine grain structure with labeled tensile specimen dimensions. In (b), laser profilometry measurements show negligible thickness variations $(<0.1 \mathrm{~mm})$ across the gauge length.

interfacial strength had a significant effect on the ability of specimens to accommodate significant plastic strains. Most specimens loaded parallel to the build height $(\mathrm{Z})$ reached the yield point but failed in a brittle manner because the foil/foil interfaces could not accommodate increasing loads. In specimens loaded in parallel with the interfaces, individual foils underwent necking, leading to eventual delamination as specimens entered the plastic instability and necking portion of the tensile curve. Individual grains near and away from interfaces were shown to be capable of handling significant additional deformation following UAM; therefore, it is hypothesized that spatially dependent foil-foil metallurgical bonding and impurities play a stronger role in UAM plate quality than effects such as UAM-induced strain accumulation and texturing.

\section{Materials and methods}

\section{Material fabrication and specimen machining}

The $\mathrm{Zr}$ plate in this work was fabricated using a $9 \mathrm{~kW}$ Soniclayer UAM unit equipped with a $350 \mathrm{M}$ steel sonotrode. A total of 25 layers of commercial-purity $200 \mu \mathrm{m}$ thick $\mathrm{Zr}$ foil of ASTM B551-12 R60702 ingot chemistry [11]) were welded atop a Zircaloy-2 baseplate to create a $3.5 \mathrm{~mm}$ tall build. A titanium
(Ti) alloy (ATI-425) foil was used as a buffer layer between the sonotrode head and the commercial-purity $\mathrm{Zr}$ foil to prevent $\mathrm{Zr}$ foil tearing during the welding process. Following plate fabrication, miniature tensile specimens were machined with gauge sections aligned with respect to the sample coordinate frame of the printed $\mathrm{Zr}$ plate. In this coordinate frame, the X-orientation was parallel to the sonotrode travel/rolling direction, the Y-orientation was parallel to the sonotrode vibration/scrubbing direction, and the Z-orientation was parallel to the build height.

The specimen type used for tensile testing was a sub-size specimen called the SS-Tiny (SST) that was originally designed for use for highly irradiated materials to reduce irradiation exposure. Owing to the dimensions of the plate fabricated using the UAM technique $(100 \times 25 \times 3.5 \mathrm{~mm})$, the SST specimen size was appropriate for the current work. A representative SST specimen fabricated using electrical discharge machining (EDM) and imaged using polarized light microscopy is illustrated in Fig. 8. Also shown for the same specimen is a thickness map projected onto the specimen surface as measured using a dual Keyence CL-3000 laser profilometry system. Although the EDM specimens may have had some minor thickness variation in the tensile head regions due to imperfections associated with 
the polishing techniques used to remove the wire-EDM surface layer, the thickness in the gauge section was uniform.

\section{Digital image correlation tensile setup}

Multiple SST specimens were tested in each orientation using an MTS Insight electromechanical test frame with a $2 \mathrm{kN}$ load cell. All tests used grips that employed shoulder loading and were performed at room temperature in air using a strain rate of $10^{-3} \mathrm{~s}^{-1}$. For many of these specimens, detailed data concerning deformation modes and strain localization/necking were collected through the DIC approach. For a detailed description of the DIC technique, as well as the pros, cons, and advances over the past decade, the reader is referred elsewhere in the literature [22].

Before testing, specimens were painted first with white paint and then with a random black speckle pattern. Then, images were collected during the tensile test at a 1 image/s frame rate using an Allied Vision GX3300 digital camera and telecentric lens. The setup allowed for a detectable resolution of $\sim 2.7$ microns/pixel. Data analysis was then performed using a combination of the commercially available VIC-2D software and a custom program that uses common 2D-DIC algorithms. The results in this work were plotted as strain rate maps, which comprised a host of information concerning active deformation processes, the spatial distribution of deformation bands, and spatially distributed strain rate data for each specimen. Following specimen failure, fractography was performed on select specimens using a TESCAN MIRA3 scanning electron microscope (SEM) operating at an accelerating voltage of $15 \mathrm{kV}$.

\section{Electron backscatter diffraction tensile setup}

EBSD specimens were prepared by first mounting and polishing one side of the SST tensile specimen to a colloidal silica finish using standard metallography techniques. The specimens were then electropolished for $4 \mathrm{~s}$ in a solution of $60 \%$ methanol, $34 \%$ 2-butoxyenthonal, and $6 \%$ perchloric acid using a voltage of $20 \mathrm{~V}$. EBSD was performed on a TESCAN MIRA3 SEM equipped with an Oxford Symmetry EBSD detector using a $30 \mathrm{kV}$ operating voltage. Analogous to the DIC tensile tests, the strain rate used for the in situ EBSD tensile test was $10^{-3} \mathrm{~s}^{-1}$. The load was reduced during EBSD scans to prevent specimen creep. Data were initially collected using the Oxford Aztec software, but data analysis was performed in EDAX OIM v8.

Data collection was performed using $2 \times 2$ binning and varying exposure times to optimize the balance between detector hit rates and scan acquisition times. Two different scan areas were used to analyze local deformation and texture evolution. For local deformation measurements, a smaller field-of-view (200 $\times 150 \mu \mathrm{m})$ was imaged using a step size of $0.5 \mu \mathrm{m}$. For texture measurements, a larger $(1200 \times 300 \mu \mathrm{m})$ scan with a $2 \mu \mathrm{m}$ step size was used to capture more grains across the gauge length and width.

\section{Acknowledgments}

This manuscript was authored by UT-Battelle LLC under Contract No. DE-AC05-00OR22725 with the US Department of Energy (DOE) and is based upon work supported by the DOE National Nuclear Security Administration, Office of Defense Nuclear nonproliferation Research and Development. The authors acknowledge the aid of Mark Norfolk and Adam Hehr at Fabrisonic LLC for their assistance with the UAM builds.

\section{Declarations}

Conflict of interest The raw data required to reproduce these findings are available upon request. The authors declare that they have no known competing financial interests or personal relationships that could have appeared to influence the work reported in this paper.

\section{Open Access}

This article is licensed under a Creative Commons Attribution 4.0 International License, which permits use, sharing, adaptation, distribution and reproduction in any medium or format, as long as you give appropriate credit to the original author(s) and the source, provide a link to the Creative Commons licence, and indicate if changes were made. The images or other third party material in this article are included in the article's Creative Commons licence, unless indicated otherwise in a credit line to the material. If material is not included in the article's Creative Commons licence and your intended use is not permitted by statutory regulation or exceeds the permitted use, you will need to obtain permission directly from the copyright holder. To view a copy of this licence, visit http://creativecommons.org/ licenses/by/4.0/.

\section{References}

1. D. White: Ultrasonic object consolidation (Google Patents, City, 2003).

2. A. Hehr, M. Norfolk, A comprehensive review of ultrasonic additive manufacturing. Rapid Prototyp. J. 26(3), 445 (2019)

3. G.J. Ram, Y. Yang, B. Stucker, Effect of process parameters on bond formation during ultrasonic consolidation of aluminum alloy 3003. J. Manuf. Syst. 25(3), 221 (2006)

4. C.M. Petrie, N. Sridharan, A. Hehr, M. Norfolk, J. Sheridan, High-temperature strain monitoring of stainless steel using fiber 
optics embedded in ultrasonically consolidated nickel layers. Smart Mater. Struct. 28(8), 085041 (2019)

5. C.M. Petrie, N. Sridharan, M. Subramanian, A. Hehr, M. Norfolk, J. Sheridan, Embedded metallized optical fibers for high temperature applications. Smart Mater. Struct. 28(5), 055012 (2019)

6. C.-H. Kuo, N. Sridharan, T. Han, M.J. Dapino, S.S. Babu, Ultrasonic additive manufacturing of 4130 steel using Ni interlayers. Sci. Technol. Weld. Join. 24(5), 382 (2019)

7. J.M. Sietins, J.W. Gillespie, S.G. Advani, Transmission electron microscopy of an ultrasonically consolidated copper-aluminum interface. J. Mater. Res. 29(17), 1970 (2014)

8. N. Sridharan, P. Wolcott, M. Dapino, S.S. Babu, Microstructure and texture evolution in aluminum and commercially pure titanium dissimilar welds fabricated using ultrasonic additive manufacturing. Scr. Mater. 117, 1 (2016)

9. A. Hehr, J. Wenning, K. Terrani, S.S. Babu, M. Norfolk, Five-axis ultrasonic additive manufacturing for nuclear component manufacture. JOM 69(3), 485 (2016)

10. C.P. Massey, C.J. Havrilak, M.N. Gussev, K.A. Terrani, A.T. Nelson, Ultrasonic additive manufacturing of zirconium: Pilot results. Mater. Lett. (under review) (2021)

11. ASTM B551/B551M, in Standard Specification for Zirconium and Zirconium Alloy Strip, Sheet, and Plate (ASTM International, City, 2017)

12. M.N. Gussev, N. Sridharan, S.S. Babu, K.A. Terrani, Influence of neutron irradiation on Al-6061 alloy produced via ultrasonic additive manufacturing. J. Nucl. Mater. 550, 152939 (2021)
13. A.G. Truog, Bond Improvement of $\mathrm{Al} / \mathrm{Cu}$ Joints Created by Very High Power Ultrasonic Additive Manufacturing (The Ohio State University, City, 2012).

14. M.N. Gussev, N. Sridharan, M. Norfolk, K.A. Terrani, S.S. Babu, Effect of post weld heat treatment on the 6061 aluminum alloy produced by ultrasonic additive manufacturing. Mater. Sci. Eng.: A 684, 606 (2017)

15. A. Akhtar, Schmid's law and prismatic slip of zirconium. Scr. Metall. 9(8), 859 (1975)

16. A. Akhtar, Prismatic slip in zirconium single crystals at elevated temperatures. Metall. Trans. A 6(6), 1217 (1975)

17. A. Akhtar, Basal slip in zirconium. Acta Metall. 21(1), 1 (1973)

18. A. Akhtar, Compression of zirconium single crystals parallel to the c-axis. J. Nucl. Mater. 47(1), 79 (1973)

19. M. Knezevic, M. Zecevic, I.J. Beyerlein, J.F. Bingert, R.J. McCabe, Strain rate and temperature effects on the selection of primary and secondary slip and twinning systems in HCP Zr. Acta Mater. 88, 55 (2015)

20. M. Yoo, Slip, twinning, and fracture in hexagonal close-packed metals. Metall. Trans. A. 12(3), 409 (1981)

21. R.J. McCabe, G. Proust, E.K. Cerreta, A. Misra, Quantitative analysis of deformation twinning in zirconium. Int. J. Plast. 25(3), 454 (2009)

22. B. Pan, Digital image correlation for surface deformation measurement: Historical developments, recent advances and future goals. Meas. Sci. Technol. 29(8), 082001 (2018) 